\title{
Calculation of the viscosity of dispersions of nanoparticles with a polymer adsorption layer in a melt
}

\author{
Tatyana Matseevich ${ }^{1 *}$, Andrey Askadskii ${ }^{1}$ \\ ${ }^{1}$ Moscow state university of civil engineering, Yaroslavskoye shosse, 26, Moscow, Russia, 129337
}

\begin{abstract}
As a result of theoretical analysis of the influence of the chemical structure of the polymer and the chemically modified surface of nanoparticles, it was shown that it is possible to predict the dependence of the viscosity of the polymer melt on the concentration of nanoparticles, their size, and the molecular weight of the polymer. Two situations have been analyzed where a strong intermolecular interaction between polymer chains and polar groups located on the surface of nanoparticles is absent, and when a strong intermolecular interaction between polymer chains and polar groups located on the surface of nanoparticles takes place.
\end{abstract}

Currently, there are a variety of models and derived equations that relate the viscosity with volume fraction of dispersed particles. One of the equations describing the dependence of the viscosity of a dispersion containing spherical particles, is Einstein's equation:

$$
\eta=\eta_{0}(1+2.5 \varphi)
$$

where $\eta$ is the viscosity of the suspension, $\eta_{0}$ is the viscosity of initial fluid, $\varphi$ is the volume of the dispersed phase, 2.5 is the parameter applicable only for spherical particles.

Another most popular equation for the concentration dependence of the viscosity of the dispersions was proposed by Mooney [1]:

$$
\eta=\eta_{0} \exp \left[\frac{2.5 \varphi}{1-\left(\varphi / \varphi^{*}\right)}\right],
$$

where $\varphi^{*}$ is the so-called critical concentration (limit value of the filling degree of solid particles). This value depends on particle shape and method of packaging, i.e. coefficient packaging. For example, the value $\varphi^{*}=0.74$ in the case of hexagonal packing of solid spherical particles.

Other popular models and the corresponding equations, we note the model of Krieger and Dougherty [2]

$$
\eta=\eta_{0}\left(1-\frac{\varphi}{\varphi^{*}}\right)^{2.5 \varphi^{*}}
$$

and model of Quemada [3]

\footnotetext{
*3atseevichTA@mgsu.ru
} 


$$
\eta=\eta_{0}\left(1-\frac{1}{2} K \varphi\right)^{-2}
$$

where $\mathrm{K}$ takes values from 2.54 to 3.71 .

Studies of concentrated suspensions $[4,5]$ belong to $\mathrm{R}$. Pal. In the works cited, there is a large amount of experimental data that can be used to test the functionality of various models describing the dependence of the relative viscosity of the suspensions in a wide concentration range.

Look at some modern models. In the work [6] the role of normal stresses in curvilinear flows of suspensions is considered. The resulting equation for describing the relative viscosity dependence on volume fraction of the dispersed phase has the form:

$$
\eta=\eta_{0}\left[1+2.5 \varphi\left(1-\frac{\varphi}{\varphi^{*}}\right)^{-1}+0.1\left(\frac{\varphi}{\varphi^{*}}\right)^{2}\left(1-\frac{\varphi}{\varphi^{*}}\right)^{-2}\right] .
$$

In the work [7] the nature of the divergence in low shear viscosity of colloidal dispersions of hard spheres is discussed. In the reviews [8-9] the analysis of the viscosity of concentrated suspensions of hard nano-size particles and bimodal spheres is provided. Proposed ratio:

$$
\eta=\eta_{0}\left(\frac{1-\varphi}{1-\frac{\varphi}{\varphi^{*}}}\right)^{\frac{2.5 \varphi^{*}}{1-\varphi^{*}}} .
$$

In [10] considers the effective viscosity of concentrated suspensions of soft particles under conditions of static and high-frequency effects. The resulting ratio is:

$$
\eta=\eta_{0}\left(1-\frac{1}{1+\frac{1}{\varphi}-\frac{1}{\varphi^{*}}}\right)^{-2.5}
$$

In the last few years, considered numerous models to describe the relative viscosity of suspensions [11-12], and in [11] proposed and proved the generalized equation for suspensions subjected to steady flow at low Reynolds numbers. For suspensions containing solid particles, the equation is written like this:

$$
\eta=\eta_{0}\left[\frac{\varphi^{*}-\varphi}{\varphi^{*}(1-\varphi)}\right]^{-\frac{2.5 \varphi^{*}}{1-\varphi^{*}}}
$$

All the considered models describe the dependence of the relative viscosity of the suspensions at different intervals of concentration of the dispersed particles depending on their volume fraction. However, no model allows one to calculate the relative viscosity of the suspension in which the dispersed particles stabilized by polymer coatings grafted on the surface of metal nanoparticles. This should be considered two options. One of them dispersed particles are inert and the other they have a strong intermolecular interaction with molecules of the liquid. In this regard, the aim of this paper is to develop a calculation scheme that allows prediction of the dependence of the volume fraction of the nanoparticles stabilized by the polymer. In the calculation scheme takes into account the chemical structure of grafted polymer. Two tasks are solved:

1) The direct problem, which allows calculating the value of relative viscosity based on chemical structure of polymer and its concentration. Such a calculation can be made 
using any of the previously proposed equations, which include the volume fraction of dispersed particles.

2) Inverse problem, which allows on the basis of measurements of the relative viscosity to estimate the size of polymer stabilized nanoparticles. Here we consider single-layer and multi-coating polymer layers of metallic nanoparticles.

For the analysis the classical Einstein (1) and Mooney (2) equations have been used. Immediately, we note that equation (1), in the same way as equation (2), in pure form, becomes inapplicable, as a result of strong intermolecular interactions between the molecules of the liquid and solid particles formed stable adsorption layer.

Consider the calculation of the dependence of viscosity on the weight fraction of nanoparticles where there is a strong intermolecular interaction between the polar groups present on the surface of the nanoparticles and the molecules of the melt. In this case, the adsorption layer formed around the nanoparticles.

The $S_{n p}$ surface of one nanoparticle is equal to

$$
S_{n p}=4 \pi R_{n p}^{2},
$$

where $R_{n p}$ is the radius of the nanoparticle. The volume of one nanoparticle is equal to

$$
v_{n p}=\frac{4}{3} \pi R_{n p}^{3} .
$$

The number of nanoparticles in the system is

$$
n_{n p}=\frac{c_{n p}}{g_{n p}},
$$

where $c_{n p}$ is the weight of nanoparticles, $g_{n p}$ is the weight of one nanoparticle. The weight of one nanoparticle is equal to

$$
g_{n p}=\rho_{n p} v_{n p}
$$

where $\rho_{n p}$ is density of nanoparticle, $v_{n p}$ is the volume of nanoparticle.

Consider $1 \mathrm{~g}$ of the systems containing polymer melt and nanoparticles. In this composition $c_{n p}$ is the weight of the nanoparticles and $1-c_{n p}$ is the weight of the solvent. Then the number of repeating units of the polymer in the composition of the $N_{c}$ is equal to

$$
N_{c}=\frac{1-c_{n p}}{M_{0}} \cdot N_{A},
$$

where $M_{0}$ is the molecular weight of the repeating unit, $N_{A}$ is Avogadro's number.

The number of repeating units per macromolecule is equal to

$$
N=\frac{M}{M_{0}}
$$

where $M$ is the molecular weight of the polymer. If the structure of the melt is globular, the volume of globule equal to

$$
V_{g l}=\frac{\left(\sum_{i} \Delta V_{i}\right)_{p} \cdot N}{k},
$$

here $\left(\sum_{i} \Delta V_{i}\right)_{p}$ is the van der Waals volume of the polymer repeating unit, $k$ is the coefficient of molecular packing. Typically for polymer melts $k=0.64$. 
The value $\left(\sum_{i} \Delta V_{i}\right)_{p}$ is calculated using a computer program "Cascade" (INEOS RAS).

The radius of the globules can be calculated using the following formula:

$$
R_{g l}=\sqrt[3]{\frac{3 V_{g l}}{4 \pi}}=\sqrt[3]{\frac{3 \sum_{i}\left(\Delta V_{i}\right)_{p} N}{4 \pi k}}
$$

The area occupied by 1 globule on the surface of the nanoparticle equal to

$$
S_{g l}=\pi R_{g l}^{2}=\pi \sqrt[3 / 2]{\frac{3\left(\sum_{i} \Delta V_{i}\right)_{p} N}{4 \pi k} .}
$$

The total surface of one nanoparticle is calculated using the formula (9). Limit the number of globules, which can be distributed on the surface of one nanoparticle is determined by the following ratio

$$
n_{g l}=\frac{S_{n p}}{S_{g l}}=\frac{4 \pi R_{n p}^{2}}{\pi \sqrt[2 / 3]{\frac{3 \sum_{i}\left(\Delta V_{i}\right)_{p} N}{4 \pi k}}}=\frac{4 R_{n p}^{2}}{0.518_{2 / 3} \sqrt{\sum_{i}\left(\Delta V_{i}\right)_{p} N}}(\text { при } k=0.64) .
$$

Taking into account formula (14) it can be written

$$
n_{g l}=\frac{7.72 R_{n p}^{2}}{\sqrt[3 / 2]{\sum_{i}\left(\Delta V_{i}\right)_{p} \frac{M}{M_{0}}}}
$$

Let's consider an example. For polyamide-6 (PA-6) the van der waals volume of the repeating unit s equal to $116 \AA^{3}, M_{0}=113$. If the radius of the nanoparticl0e is equal to $50 \AA$ $(5 \mathrm{~nm})$ and the molecular weight of the polymer $M=14000$, the value of $n_{g l}$, calculated by the formula (19), equal to 32.6.

It is obvious that the radius of one nanoparticle together with the adsorption layer is equal to:

$$
R_{n p+a d . l .}=R_{n p}+2 R_{g l}=R_{n p}+2 \sqrt[3]{\frac{3\left(\sum_{i} \Delta V_{i}\right)_{p} N}{4 \pi k}}=R_{n p}+1.44 \sqrt[3]{\left(\sum_{i} \Delta V_{i}\right)_{p} \frac{M}{M_{0}}} .
$$

The volume of single nanoparticle with adsorptive layer is

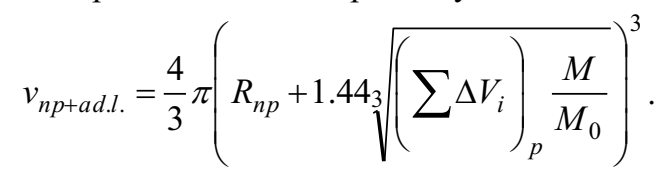

For nanoparticles that do not contain adsorption layers, their volume fraction can be calculated by the following formula

$$
\varphi=\frac{v_{n p} n_{n p}}{v_{n p} n_{n p}+V_{p}}
$$

where $V_{p}$ is the volume of polymer in the composition.

For nanoparticles containing adsorption layers, their volume fraction can be calculated using the following formula 


$$
\varphi_{n p+a d . l .}=\frac{v_{n p+a d . l .} n_{n p}}{v_{n p+a d . l .} n_{n p}+V_{p}-v_{\text {ad.l. }} n_{n p}},
$$

where $v_{a d . l}$. is the volume of the adsorption layer per one nanoparticle,

$$
V_{p}=\frac{G_{p}}{\rho_{p}},
$$

where $G_{p}$ is the weight of the polymer in the composition, and $\rho_{p}$ is the density of the polymer.

If we analyze $1 \mathrm{~g}$ of the composition, $G_{p}=1-c_{n p}$. The density of the polymer is calculated by the following formula [13]:

$$
\rho_{p}=\frac{k M_{0}}{N_{A}\left(\sum_{i} \Delta V_{i}\right)_{p}} .
$$

Thus, we obtain

$$
V_{p}=\frac{\left(1-c_{n p}\right) N_{A}\left(\sum_{i} \Delta V_{i}\right)_{p}}{k M_{0}} .
$$

The volume of the adsorption layer of $V_{\text {ad.l. }}$ is:

$$
V_{\text {ad.l. }}=n_{g l} \cdot V_{g l} \text {. }
$$

Substituting relations (15) and (19) into formula (27), we get:

$$
V_{\text {ad.l. }}=\frac{7.72 R_{n p}^{2} \sqrt[3]{\left(\sum_{i} \Delta V_{i}\right)_{p}}}{k} .
$$

Substituting formula (11), (12), (26) and (28) into relation (23), we can write the relation for the volume fraction of nanoparticles containing adsorption layers:

$$
\varphi_{n p+\text { ad.l. }}=\frac{\left(R_{n p}+1.44 \sqrt[3]{\left.\frac{M}{M_{0}}\left(\sum_{i} \Delta V_{i}\right)_{p}\right)^{3} \frac{c_{n p}}{\rho_{n p} R_{n p}^{3}}}\right.}{\left(R_{n p}+1.44 \sqrt[3]{\frac{M}{M_{0}}\left(\sum_{i} \Delta V_{i}\right)_{p}}\right)^{3} \frac{c_{n p}}{\rho_{n p} R_{n p}^{3}}+\frac{\left(1-c_{n p}\right) N_{A}\left(\sum_{i} \Delta V_{i}\right)_{p}}{k M_{0}}-\frac{7.72 \sqrt[3]{\left(\sum_{i} \Delta V_{i}\right)_{p}} \cdot 3 c_{n p}}{4 \pi \rho_{n p} R_{n p}}}
$$

Now consider the examples of calculations.

The initial polymer is polyamide- 6 . The parameters of the polymer system are given in table 1 .

Table 1. Parameters for the system "PA-6 + nanoparticles".

\begin{tabular}{|l|c|}
\hline Molecular weight of the repeating units, $M_{0}$ & 113 \\
\hline Molecular weight of the polymer, $M$ & 14000 \\
\hline Van-der-Waals volume of the repeating units of the polymer, & 116 \\
$\left(\sum_{i} \Delta V_{i}\right)_{p}, \AA^{3}$ & \\
\hline
\end{tabular}




\begin{tabular}{|l|c|}
\hline Coefficient of molecular packing for melt, $k$ & 0.64 \\
\hline Weigh of the nanoparticles containing in 1 g of composition, g & 0.1 \\
\hline Radius of nanoparticle $R_{n p}, \AA$ & 50 \\
\hline Density of nanoparticle $\rho_{n p}, \mathrm{~g} / \mathrm{cm}^{3}$ & 1.54 \\
\hline Zero shear viscosity for PA-6 at $463 \mathrm{~K}, \mathrm{~N} \cdot \mathrm{sec} / \mathrm{cm}^{2}$ & 15.2 \\
\hline
\end{tabular}

If the adsorption layer is absent, the volume fraction of nanoparticles is calculated using the following formula

$$
\varphi=\frac{\frac{c_{n p}}{\rho_{n p}}}{\frac{c_{n p}}{\rho_{n p}}+\frac{1-c_{n p}}{\rho_{p}}}=\frac{\frac{c_{n p}}{\rho_{n p}}}{\frac{c_{n p}}{\rho_{n p}}+\frac{\left(1-c_{n p}\right) N_{A}\left(\sum_{i} \Delta V_{i}\right)_{p}}{k M_{0}}} .
$$

Substituting all the parameters available in the table 1 , into formula (30), obtain $\varphi=$ 0.0695 .

If the surface of the particles formed adsorption layer, using all parameters from table 1 and formula (29), have $\varphi_{n p+a d . l}=0.2226$.

Now determine the dependence of viscosity on the weight fraction of nanoparticles. The type of this dependence is determined by the possibility of the formation of a polymeric adsorption layer on the surface of the nanoparticles.

1 - There is no a strong intermolecular interaction between polymer chains and the polar groups on the surface of nanoparticles. Then, substituting all the system parameters from table 1 into equation (1), we obtain

$$
\eta=15.2\left(1+\frac{1.6225 c_{n p}}{0.966-0.317 c_{n p}}\right) .
$$

2 - There is a strong intermolecular interaction between polymer chains and polar groups localized at the surface of nanoparticles. Then we get the following formula

$$
\eta=15.2\left(1+\frac{7.9763 c_{n p}}{2.0419 c_{n p}+0.9661}\right) .
$$

Next, let's calculate these dependencies using equation Mooney (2). When the strong intermolecular interaction between polymer chains and the polar groups on the surface of nanoparticles is absent, substituting all the parameters from table 1 into equation (1) with $\varphi^{*}=0.74$ (hexagonal packaging), we get:

$$
\eta=15.2 \exp \frac{1.6225 c_{n p}}{0.966-1.194 c_{n p}} .
$$

When a strong intermolecular interaction is manifested, we obtain the following formula:

$$
\eta=15.2 \exp \frac{7.9763 c_{n p}}{0.966-2.2695 c_{n p}} .
$$

The dependence of viscosity on concentration of nanoparticles calculated by the Einstein equation (1) shown in figure 1. The dependencies of viscosity on concentration of nanoparticles calculated by Mooney equation (2) shown in figure 2 . 


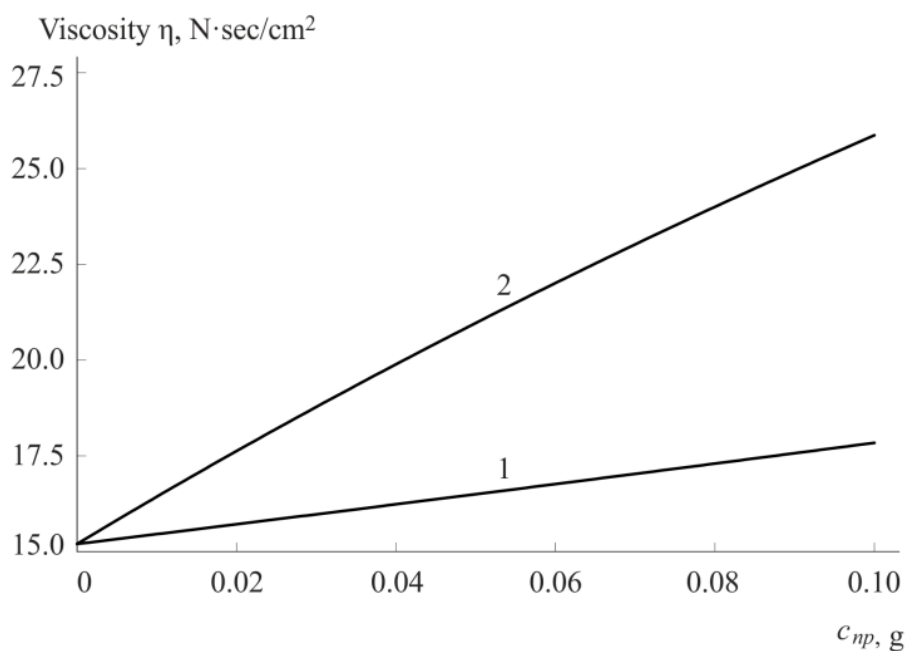

Fig. 1. Dependencies of viscosity on the weight of nanoparticles in $1 \mathrm{~g}$ of composite containing PA-6 and nanoparticles. 1 - There is no strong intermolecular interaction between polymer chains and polar groups located on the surface of nanoparticles. 2 - Strong intermolecular interaction between polymer chains and polar groups located on the surface of nanoparticles takes place. The calculations were performed using Einstein equation (1).

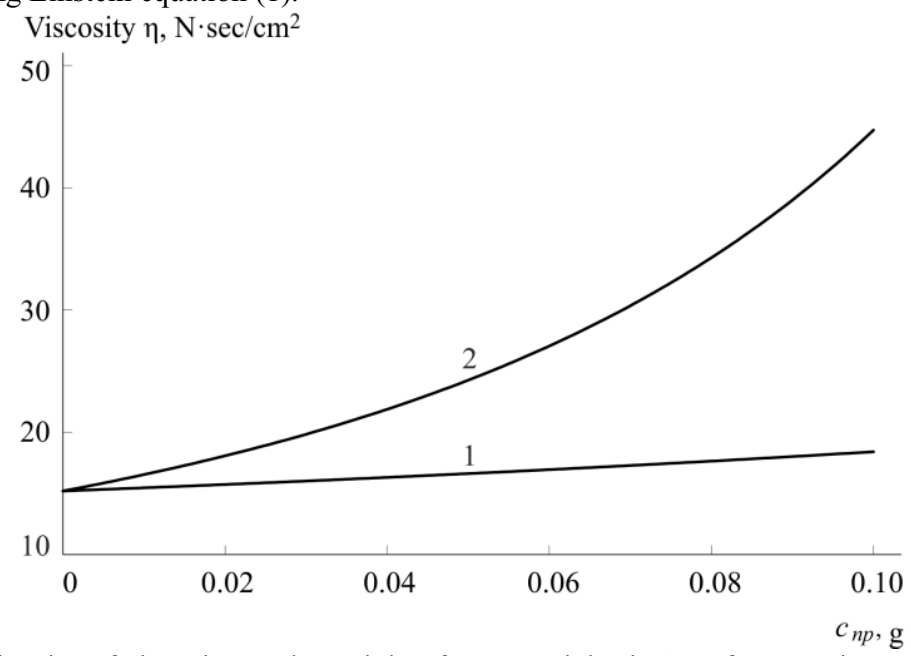

Fig. 2. Dependencies of viscosity on the weight of nanoparticles in $1 \mathrm{~g}$ of composite containing PA-6 and nanoparticles. 1 - There is no strong intermolecular interaction between polymer chains and polar groups located on the surface of nanoparticles. 2 - Strong intermolecular interaction takes place. The calculations were performed using Mooney equation (2).

Now let's calculate the dependencies of the viscosity on the radius of nanoparticles. When the adsorption layer is absent, the viscosity is independent of the size of nanoparticles. If the adsorption layer is formed, the relations for description of the dependencies of viscosity on the radius of nanoparticles are as follows (taking into account the values of all parameters are available in table 1). The dependencies of viscosity on radius of nanoparticles are described by relations (35) and (36).

The dependencies of the viscosity on the radius of nanoparticles are shown in figure 3 . It can be seen that in agreement with equation Mooney the viscosity increases sharply when the size of nanoparticles decreases. 


$$
\begin{aligned}
& \eta=15.2\left(1+\frac{0.16225\left(\frac{R_{n p}+35}{R_{n p}}\right)^{3}}{0.0649\left(\frac{R_{n p}+35}{R_{n p}}\right)^{3}+0.8685-\frac{0.912}{R_{n p}}}\right) \text {, (Einstein equation) (35) } \\
& \eta=15.2 \exp \left(\frac{0.16225\left(\frac{\left.R_{n p}+35\right)}{R_{n p}}\right)^{3}}{0.8695-\frac{0.912}{R_{n p}}-0.0228\left(\frac{\left.R_{n p}+35\right)}{R_{n p}}\right)^{3}}\right) \cdot \text { (Mooney equation) (36) }
\end{aligned}
$$

Viscosity $\eta, N \cdot \sec / \mathrm{cm}^{2}$

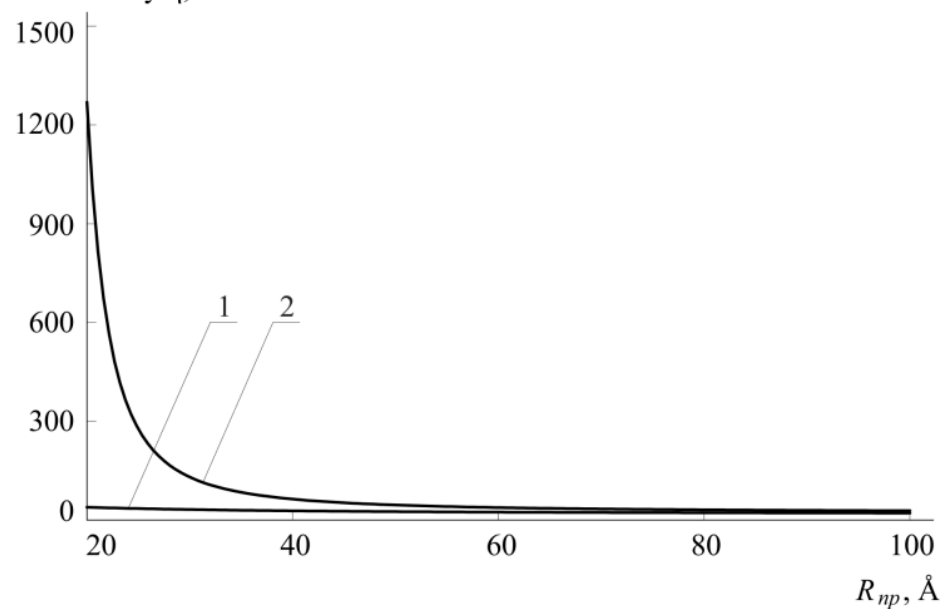

Fig. 3. Dependence of viscosity on the radius of nanoparticles. 1 - The viscosity is calculated by Einstein equation; 2 - The viscosity is calculated by Mooney equation. The adsorption layer takes place.

\section{References.}

1. M. Moony, J. Colloid. Interface Sci. 6, 2, 162 (1951)

2. I.M. Krieger, T.J. Dougherty, Trans. Soc. Rheol. III, 137 (1959)

3. D. Quemada, Rheol. Acta 16, 82 (1977)

4. R. Pal, Ind. Eng. Chem. Res. 43, 17, 5372 (2004)

5. R. Pal, J. Biomech. 36, 7, 981 (2003)

6. J.F. Morris, F. Boulay, J. Rheol. 43, 5, 1213 (1999)

7. Z. Cheng, J. Zhu, P.M. Chaikin, S.E. Phan, W.B. Russel, Phys. Rev. 65, 4, 041405-1 (2002)

8. H.J.H. Brouwers, Phys. Rev. 5, 051402-1 (2010)

9. H.J.H. Brouwers, Phys. Rev. 3, 032202-1 (2013)

10. C.I. Mendoza, J. Chem. Phys. 135, 054904-1 (2011)

11. S.A. Faroughi, C. Huber, Rheol. Acta. 54, 85 (2015)

12. A. Dörr, A. Sadiki, A. Mehdizadeh, J. Rheol. 57, 3, 743 (2013)

13. A.A. Askadskii, Computational Materials Science of Polymers (CISP, 2003) 